\title{
DIVERSIDAD, HÁBITO Y HÁBITAT DE MACRÓFITOS ACUÁTICOS EN LA PATAGONIA OCCIDENTAL (REGIÓN DE AISÉN, CHILE)
}

\author{
DIVERSITY, HABIT AND HABITAT OF AQUATIC VASCULAR MACROPHYTES \\ OF THE WESTERN PATAGONIA (AISÉN REGION, CHILE)
}

Cristina San Martín ${ }^{1}$, Yessica Pérez ${ }^{1}$, Drina Montenegro ${ }^{1} \&$ Miguel Álvarez $^{2}$

\begin{abstract}
We studied the abundance, phytogeographycal origin, biological spectrum and the habitats of the aquatic macrophyte flora in the limnic (lotic and lentic) wetlands and in salt marshes of the Aisén Region, Chile. We found 86 species of vascular macrophytes including in 54 genera and 33 families. Only 8 species are abundant (Myriophyllum quitense, Potamogeton stenostachys, Glyceria multiflora, Hippuris vulgaris, Marsippospermum grandiflorum, Tetroncion magellanicum, Scirpus californicus and Gunnera magellanica), most of them occurring in swampy habitats from the river banks. Of the total species number, 73 are native and 13 introduced, indicating little human disturbance in those wetlands. The biological spectrum consists of 54 perennial herbs (hemicryptophytes) and 23 tall herbs with underwater rhizomes and aerial culms (cryptophytes). The tall perennial herbs (chamaephytes) are represented by 6 species and the annuals (therophytes) by 3 . Sixty seven swampy emergent forms (helophytic) and 19 species of aquatic plants (submerged, with floating leaves and free floating hydrophytes) were collected. The wetlands with higher species richness were the lowland ponds with 44 species and streams with 37 species. Small stands of coastal marshes were the only brackish habitat studied here, containing 5 halophytes (Cotula coronopifolia, Puccinellia glaucescens, Triglochin concinna, Ruppia filifolia y Zannichellia palustris). We analyzed the historical development of the knowledge of the aquatic macrophytes in the Aisén Region, concluding that the most of the species had been prospected before. Only Veronica Anagallis-aquatica and Lycopus europaeus, can be considered as newly introduced neophytes, being the first an invasive species. Finally, we discuss the results compared with those found in aquatic environments in other water bodies from northern parts of central Chile.
\end{abstract}

Key words: Aquatic plants, vascular macrophytes, biodiversity, Chilean Patagonia. 


\section{RESUMEN}

Se estudió la abundancia, el origen, el espectro biológico y los hábitats de la flora de macrófitos acuáticos en los cuerpos límnicos (lóticos y lénticos) y en las marismas salobres de la Patagonia occidental de la Región de Aisén, Chile. Se encontraron 86 especies de macrófitos vasculares incluidos en 54 géneros y 33 familias. Sólo 8 especies son abundantes (Myriophyllum quitense, Potamogeton stenostachys, Glyceria multiflora, Hippuris vulgaris, Marsippospermum grandiflorum, Tetroncion magellanicum, Scirpus californicus y Gunnera magellanica) la mayoría de ellas ocupando los hábitats que ofrecen los abundantes pantanos ribereños. Del total de especies 73 son autóctonas y sólo 13 son alóctonas, lo que señala escasa intervención humana en estos cuerpos acuáticos. El espectro biológico está integrado por 54 hierbas perennes (hemicriptófitos) y 23 hierbas grandes con rizomas subacuáticos y culmos aéreos (criptófitos). Hierbas erguidas y subarbustos (caméfitos) presentaron 6 especies y las hierbas anuales (terófitos) 3. De las formas palustres emergidas (helófitos) se colectaron 67 especies y de las acuáticas (sumergidas, natantes y flotantes libre) 19. Los cuerpos acuáticos con mayor riqueza florística fueron las lagunas de tierras bajas con 44 especies y esteros con poca corriente con presencia de 37 especies. Pequeños rodales de marismas litorales fueron los únicos ambientes salobres encontrados y, en ellos, se colectaron 5 halófitos (Cotula coronopifolia, Puccinellia glaucescens, Triglochin concinna, Ruppia filifolia y Zannichellia palustris). Además se analizó el desarrollo histórico del conocimiento que se tiene de la flora acuática macrofítica de la región de Aisén, concluyendo que la mayoría de las especies encontradas había sido prospectada con anterioridad. Sólo Verónica anagallis-aquatica y Lycopus europaeus, pueden considerarse neófitos de reciente introducción, la primera con carácter de invasora. Por último, se discuten los resultados comparándolos con los encontrados en ambientes acuáticos de otras regiones más septentrionales de Chile central.

Palabras clave: Plantas acuáticas, macrófitos vasculares, biodiversidad, Patagonia chilena.

\section{INTRODUCCION}

La Región de Aisén del General Carlos Ibáñez del Campo (Chile) es una región aislada, aún de escasa conectividad con el resto del país (Rojas \& Leidich 2006) y, por ello, con una baja densidad poblacional y una ganadería que, aunque extensiva, ejerce una fuerte presión de cambio de uso en los bosques, los que son transformados en praderas o en silvopastoreo (Vidal et al. 2011). Producto de ello, el cambio de uso del suelo y la introducción de malezas, impiden la regeneración de la vegetación boscosa (Ramírez et al. 1996). Además, la rigurosidad del clima exige el uso de calefacción doméstica de manera intensa, lo que representa una fuerte presión de uso sobre los bosques (Murúa et al. 1993). Otro factor importante es el consumo de madera para construcción sin condiciones silviculturales, lo cual degrada los bosques de Nothofagus pumilio (Lenga) $y$, especialmente, las poblaciones vulnerables de Pilgerodendron uviferum (Ciprés de las Guaitecas) (Rovere et al. 2002). De esta manera urge conocer las características de los ecosistemas de esta región para planificar un manejo racional, que será muy necesario a futuro cuando, al mejorar la conectividad, aumente la población y los efectos antrópicos negativos se multipliquen.

También el cambio climático está afectando la ecología y el equilibrio ambiental de la Región de Aisén, ya que el retroceso de los glaciares disminuye las reservas de agua, fenómeno que ha sido estudiado en detalle por Fernández et al. (2006). Los fenómenos "GLOF" (Glacial Lake Outburst Floods) están variando el régimen de crecidas de los ríos y éstas se están haciendo cada año más frecuentes (CECS 2008 ${ }^{1}$, Rojas \& Leidich 2009). Junto con lo anterior, se ha observado sequedad en turberas esfagnosas y reemplazo de Sphagnum magellanicum por Marsippospermum grandiflorum (Villagra et

1 Centro de Estudios Científicos del Sur (CECS). 2008. Lago Cachet 2 vuelve a "vaciarse" en Patagonia. Comunicado de Prensa del 10.10.08. (http://www.glaciologia.cl/colonia. html). 
al. 2009), aumento de líquenes epifíticos, muerte de líquenes terrestres, sequía en especies de flora $e$ incluso muerte de bosques de Lenga (observaciones no publicadas de los autores). Todo lo anterior demanda una mayor preocupación por los estudios biológicos, hídricos y climáticos.

La mejor manera de planificar su protección es conocer la naturaleza y las características de estos cuerpos de agua, cuyas cuencas muestran una gran dinámica (Mardones et al. 2007). Un componente importante de estos ecosistemas son los macrófitos acuáticos que allí forman hábitats para otros organismos. En ese contexto, el presente trabajo pretende ser una contribución al conocimiento de los macrófitos acuáticos que colonizan dichos ambientes, dada la dispersión de la literatura y la ausencia de revisiones sobre esta flora para la $\mathrm{Re}^{-}$ gión de Aisén. La abundancia de estos ambientes permite suponer a priori la presencia de una gran diversidad de plantas acuáticas en la región, aunque el clima más frío, reduciría la diversidad de formas y el tamaño de las poblaciones (Ramírez et al. 1986).

\section{MATERIAL Y MÉTODOS}

Desde el año 2000 hasta la fecha y con frecuentes visitas, se realizaron exhaustivas colectas de plantas hidrófilas (acuáticas y palustres) en la gran variedad de ambientes acuáticos existentes en la Región de Aisén del General Carlos Ibáñez del Campo, Chile, incluyendo algunas islas del litoral, lo que permitió compilar un catálogo de la flora hidrófila de esa región (Fig. 1). Los ejemplares colectados se determinaron utilizando la literatura disponible

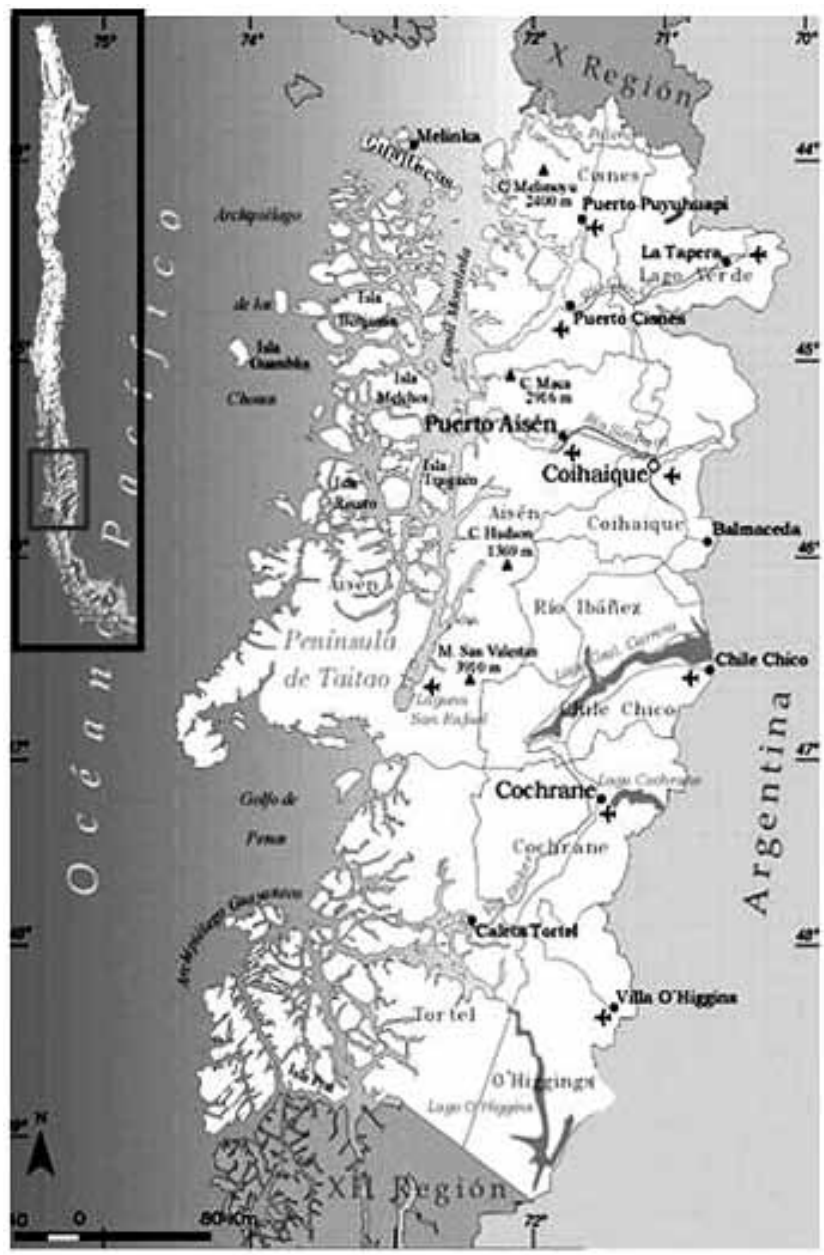

Fig. 1. Región de Aisén, Patagonia chilena. 
(Arber 1963, Cook et al. 1974, Hutchinson 1975, Ramírez et al. 1982, Rodríguez \& Dellarossa 1998). La nomenclatura sigue a Marticorena \& Quezada (1985) aunque se agregaron cambios nomenclaturales más actuales (Matthei 1995, Ramírez \& San Martín 2006a). De la misma manera, la nomenclatura de Pteridófitos sigue a Rodríguez (1995). Para la clasificación de las angiospermas se usó la información de la página "Angiosperm Phylogeny Website" (http:// www.mobot.org/mobot/research/apweb), administrada por el Missouri Botanical Gardens, mientras que para la clasificación de pteridófitos se usó como referencia "USDA Plants Database" (http://plants. usda.gov). Dicho esquema se detalla en la Tabla 1.

Dado el carácter autóctono o alóctono de las especies se estableció que en base a Ramírez \& San Martín (2006a, 2006c) la proporción de estos dos elementos en la flora permitirán extraer conclusiones respecto del grado de alteración antrópica actual presentada por los cuerpos acuáticos estudiados (Frey \& Lösch 1998).

Para la clasificación del cuerpo vegetativo de las plantas colectadas se utilizaron las formas de vida de Raunkiaer que incluyen fanerófitos, caméfitos, hemicriptófitos, criptófitos y terófitos, revisadas por Mueller-Dombois \& Ellenberg (1974). Las formas de crecimiento específicas de plantas acuáticas se tomaron de la clasificación de Ramírez \& Stegmaier (1982) actualizada en Ramírez \& San Martín (2006a), la que diferencia entre helófitos (plantas palustres) $e$ hidrófitos (plantas acuáticas). Los últimos se clasificaron en sumergidas, natantes y flotantes libres. Con las formas de vida se determinaron los espectros biológicos, que entregan referencias respecto de las características físicas de los cuerpos acuáticos estudiados.

También se ingresó en la lista de las especies los valores indicadores para luz, temperatura, reacción del suelo $(\mathrm{pH})$, contenido de nitrógeno aprovechable en el suelo, y humedad, con el fin de confeccionar espectros indicadores. Dichos valores se obtuvieron del trabajo de San Martín et al. (2003) y se expresan con la escala de 1 a 9 propuesta por Ellenberg (1979) y utilizada recientemente en Chile por San Martín et al. (2009).

Los cuerpos acuáticos fueron clasificados de acuerdo a las indicaciones de Campos (1979) diferenciando entre lóticos y lénticos y se completaron con las clasificaciones de humedales, propuestas por
Tabla 1. Esquema de clasificación taxonómica usada en este trabajo.

\begin{tabular}{|c|c|}
\hline Grupo & Grupo \\
\hline Orden & Orden \\
\hline Familia & Familia \\
\hline Dicotiledóneas & Monocotiledóneas \\
\hline Apiales & Alismatales \\
\hline Apiaceae & Araceae \\
\hline Araliaceae & Juncaginaceae \\
\hline Asterales & Potamogetonaceae \\
\hline Asteraceae & Ruppiaceae \\
\hline Campanulaceae & Poales \\
\hline Brassicales & Cyperaceae \\
\hline Brassicaceae & Juncaceae \\
\hline Caryophyllales & Poaceae \\
\hline \multicolumn{2}{|l|}{ Polygonaceae } \\
\hline Portulacaceae & Pteridófitas \\
\hline Dipsacales & Equisetales \\
\hline Valerianaceae & Equisetaceae \\
\hline Ericales & Hydropteridales \\
\hline Primulaceae & Azollaceae \\
\hline Gunnerales & Isoetales \\
\hline Gunneraceae & Isoetaceae \\
\hline Lamiales & Polypodiales \\
\hline Calceolariaceae & Blechnaceae \\
\hline \multicolumn{2}{|l|}{ Lamiaceae } \\
\hline \multicolumn{2}{|l|}{ Phrymaceae } \\
\hline \multicolumn{2}{|l|}{ Plantaginaceae } \\
\hline \multicolumn{2}{|l|}{ Scrophulariaceae } \\
\hline \multicolumn{2}{|l|}{ Malpighiales } \\
\hline \multicolumn{2}{|l|}{ Euphorbiaceae } \\
\hline \multicolumn{2}{|l|}{ Myrtales } \\
\hline \multicolumn{2}{|l|}{ Lythraceae } \\
\hline \multicolumn{2}{|l|}{ Onagraceae } \\
\hline \multicolumn{2}{|l|}{ Ranunculales } \\
\hline \multicolumn{2}{|l|}{ Ranunculaceae } \\
\hline \multicolumn{2}{|l|}{ Rosales } \\
\hline \multicolumn{2}{|l|}{ Rosaceae } \\
\hline \multicolumn{2}{|l|}{ Saxifragales } \\
\hline \multicolumn{2}{|l|}{ Crassulaceae } \\
\hline Haloragaceae & \\
\hline
\end{tabular}

Ramsar (Davis et al. 1996) y Ramírez et al. (2002). Con lo anterior, se estableció una clasificación de cuerpos acuáticos que permite incluir todos los ambientes acuáticos límnicos (dulciacuícolas) y salobres estudiados en esta investigación y analizar la flora específica de cada grupo. También se diferenció entre cuerpos acuáticos límnicos y salobres, estos últimos representados únicamente por pequeños rodales de marismas litorales colonizadas por 
halófitos muy especializados (Álvarez et al. 2010, Ramírez et al. 1989).

La composición florística de los distintos tipos de humedales fue comparada a través de una ordenación "nMDS (Escalamiento multidimensional no métrico). Para ello se calculó una matriz de distancia usando la presencia de las especies en los distintos tipos de hábitats y el índice de Jaccard, restringiendo la ordenación a dos dimensiones. También se superpuso la posición de las especies calculando sus centroides en el diagrama de ordenación (Lepš \& Šmilauer 2003, Leyer \& Wesche 2007). Además, se calcularon modelos de regresión logística (Menard 2002) entre las coordenadas del nMDS y las características de cada especie. Dichos modelos fueron comparados usando el índice de Akaike (AIC: Akaike's Information Criterion).

Por último, se compararon los resultados de este trabajo con aquellos presentados por las distintas prospecciones de flora acuática y terrestre realizada por varios autores desde 1935 a la actualidad en la Región de Aisén. Esto permitió establecer una revisión histórica del conocimiento de las plantas acuáticas en la región y la presencia de neófitos que pudiesen transformarse a futuro, en posibles invasores.

\section{RESULTADOS}

La Región de Aisén del General Carlos Ibáñez del Campo posee una variada gama de ambientes acuáticos, en los cuales y, debido a su gran diversidad, es posible encontrar muchas especies de macrófitos hidrófilos. En estos hábitats se encontró un total de 86 especies de plantas acuáticas y palustres distribuidas en 19 órdenes (Tabla 1). El grupo de especies mejor representado fueron las dicotiledóneas, con 50 especies, lo cual equivale a un 58,14\% del total. Las monocotiledóneas están representadas por 32 especies, es decir, 37,21\% del total, mientras que los pteridófitos solamente por 4 especies (Azolla filiculoides, Blechnum chilense, Equisetum bogotense e Isoetes savatieri), alcanzando un 4,65\% del número total de especies. Un completo catálogo de la flora acuática y palustre de Aisén se presenta en el anexo 1.

Las especies prospectadas se agrupan en 33 familias y 54 géneros. El número promedio de especies por familia fue de 2,6, siendo la fa- milia Cyperaceae, con 12 representantes, la más numerosa. Le siguen en importancia las familias Plantaginaceae y Ranunculaceae, con 9 especies cada una, y Juncaceae, con 7. En el histograma de la Fig. 2 se puede observar que no existen familias con los rangos de 8,10 y 11 especies. Los rangos de 3, 4, 5, 6, 9 y 12 especies, poseen solamente una familia en cada caso. Más frecuentes son las 19 familias con sólo una especie, representando un 57,58 $\%$ del total de familias. Finalmente, con 2 especies existen 6 familias (Brassicaceae, Campanulaceae, Gunneraceae, Onagraceae, Potamogetonaceae y Rosaceae) y con 9 especies 2 (Plantaginaceae y Ranunculaceae).

El promedio de especies por género fue de 1,6 , siendo los géneros más importantes Ranunculus, con 8 especies, junto a Carex y Juncus, con 6 especies cada uno. Por último, la cantidad promedio de géneros por familia fue de 1,6, siendo las familias Plantaginaceae y Poaceae las que presentaron más géneros (5 cada una), seguidas por Asteraceae y Cyperaceae, ambas con 4 géneros cada una, y por Juncaginaceae, con 3. Las restantes familias, menos diversas, poseen de 1 a 2 géneros.

Muy pocas especies acuáticas del catálogo presentan grandes poblaciones, entre ellas, la hierba sumergida Myriophyllum quitense y las especies natantes Potamogeton stenostachys y Glyceria multiflora que crecen en ambientes lóticos, a veces con bastante corriente. Hippuris vulgaris es una

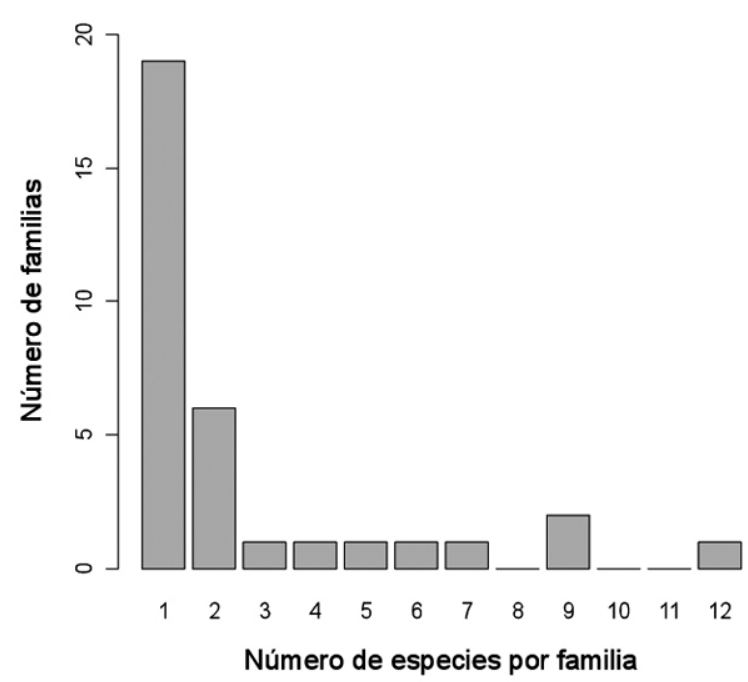

Fig. 2. Histograma de frecuencia de las especies por familias. 
especie introducida abundante en ambientes lénticos, especialmente lagunas de pequeño tamaño donde crece asociado a un alga Carácea del género Nitella. Esta especie es la única que está restringida al extremo Sur del país, es decir, Regiones de Aisén y Magallanes. Marsippospermum grandiflorum y Tetroncion magellanicum son muy abundantes en lagunas y arroyos de turberas esfagnosas. Scirpus californicus forma grandes poblaciones en lagunas de tierras bajas, pero también se presenta en altura, aunque con menor abundancia. Por último, cabe mencionar a Gunnera magellanica especie muy frecuente y abundante en ambientes con menor anegamiento, incluso terrestres.

Según su origen fitogeográfico, las especies se pueden agrupar en nativas e introducidas. No obstante, como las plantas acuáticas constituyen una flora de tipo azonal, es decir, que no depende tanto del macroclima, sino del agua del suelo, las especies presentan áreas de distribución muy amplias, incluso cosmopolitas, por lo que es muy difícil establecer el origen fitogeográfico con toda certeza para cada caso. El mayor porcentaje de abundancia lo obtuvieron las plantas nativas con 73 especies $(84,88 \%)$, mientras que las malezas introducidas solo alcanzan al 15,12\% de la flora total con 13 especies (Tabla 2). Como la presencia de elementos alóctonos se corresponde positivamente con el grado de alteración antrópica de un lugar, se puede concluir que el grado de disturbio de los hábitats acuáticos estudiados, especialmente de los límnicos, es aún reducido (Hauenstein et al. 1996).

El espectro biológico de la flora de los cuerpos acuáticos descritos es incompleto, faltando en él los fanerófitos leñosos que tienen representantes palustres, principalmente en los trópicos. Aunque las Coniferas nativas Lepidothamnus fonckii y Pilgerodendron uviferum podrían considerarse plantas leñosas palustres, no se incluyeron en esta lista por colonizar ambientes turbosos no estudiados en este trabajo. De las especies encontradas, 54 corresponden a hierbas perennes hemicriptofíticas, lo que representa un $62,79 \%$, del total, siendo la forma de mayor abundancia (Tabla 2 y Fig. 3). En segundo lugar están los criptófitos (hierbas grandes con culmos aéreos de corta vida y órganos subterráneos perdurantes) con 23 especies (26,74\%), éstos son comunes en lugares palustres de sustrato inestable y con anegamiento fluctuante, que corresponden a situaciones desfavorables incluso para los helófitos.

Tabla 2. Diversidad de los macrófitos a distintos niveles taxonómicos y su distribución en los grupos funcionales.

\begin{tabular}{|c|c|c|c|c|}
\hline & Especies & Géneros & Familias & Órdenes \\
\hline Taxa totales & 86 & 54 & 33 & 19 \\
\hline \multicolumn{5}{|l|}{ Grupos vegetales } \\
\hline pteridófitas & 4 & 4 & 4 & 4 \\
\hline dicotiledóneas & 50 & 32 & 22 & 13 \\
\hline monocotiledóneas & 32 & 18 & 7 & 2 \\
\hline \multicolumn{5}{|l|}{ Origen } \\
\hline nativas & 73 & 45 & 30 & 19 \\
\hline introducidas & 13 & 13 & 10 & 7 \\
\hline \multicolumn{5}{|c|}{ Formas de vida según Ramírez \& Stegmeier } \\
\hline helófitas & 68 & 40 & 23 & 16 \\
\hline natantes & 4 & 4 & 3 & 3 \\
\hline flotantes libres & 2 & 2 & 2 & 2 \\
\hline submersas & 12 & 10 & 10 & 7 \\
\hline \multicolumn{5}{|c|}{ Formas de vida según Raunkiaer } \\
\hline caméfitas & 6 & 4 & 4 & 2 \\
\hline hemicriptófitas & 54 & 31 & 18 & 14 \\
\hline criptófitas & 23 & 20 & 17 & 12 \\
\hline terófitas & 3 & 3 & 3 & 3 \\
\hline \multicolumn{5}{|c|}{ Salinidad preferencial } \\
\hline dulce & 71 & 43 & 30 & 18 \\
\hline salobre & 5 & 5 & 5 & 3 \\
\hline ambas & 10 & 9 & 8 & 7 \\
\hline
\end{tabular}




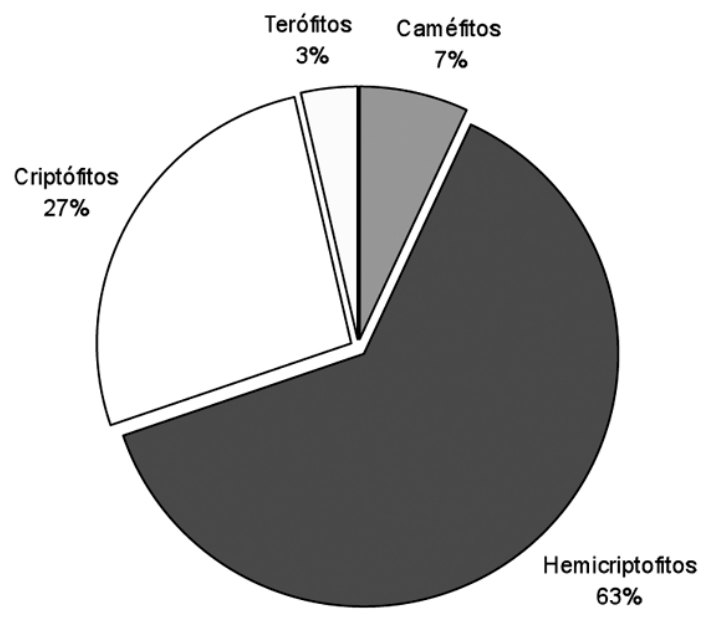

Fig. 3. Distribución del número de especies por forma de vida.

Escasamente representadas se encuentran las formas camefíticas (hierbas erguidas) con 6 especies y 6,97 $\%$ y los terófítos o hierbas anuales con 3 especies y 3,5\%. Este bajo número de especies se debe principalmente, a que esta forma de vida es típica de ambientes con sequía. Este espectro biológico corresponde a lo que es dable esperar en la flora de los ambientes dulciacuícolas estudiados.

De acuerdo al tipo de anegamiento del ambiente acuático que colonizan los macrófitos hidrófilos se pueden separar en hidrófitos y helófitos. Los primeros corresponden a aquellos que dependen del anegamiento para subsistir y, por ello, incluyen las formas: sumergidas (viven mayoritariamente bajo la superficie, no obstante en primavera suelen emerger sus flores), natantes (arraigadas al sustrato, poseen hojas sumergidas y otras que flotan en superficie) y flotantes libres (flotan libremente sobre la superficie, sin arraigo al sustrato). Estos grupos en conjunto, presentaron 18 especies, aportando con sólo el 20,93 \% del total (Tabla 2). Los helofitos palustres, considerados tambien plantas acuáticas emergidas, son los mejor representados con el 79,07 \% y 68 especies. Esta situación se corresponde con la abundancia de hábitats para esta forma de vida en el gradiente ribereño, donde el diferente nivel de anegamiento y la depositación de sedimentos condicionan variaciones de los biótopos en espacios muy reducidos, haciéndolos muy diversos. Por lo demás, son precisamente los ambientes palustres ribereños los colonizados por los macrófitos y no los pelágicos, donde el arraigamiento está limitado por la mayor profundidad y la corriente.

Las 18 especies de hidrófitos se clasificaron en 12 especies sumergidas, 4 natantes y sólo 2 flotantes libres (Azolla filiculoides y Lemna minima), de menor abundancia (Tabla 2 y Fig. 4). Las primeras colonizan aguas transparentes y oligotróficas, bajo condiciones climáticas más adversas, ya que ellas logran una mayor protección al sumergirse en el medio acuático. Las especies natantes poseen dimorfismo foliar y, por ello, al aumentar mucho la corriente en épocas de mayor escorrentía o de bajas temperaturas, pueden sobrevivir desprendiéndose de su hojas natantes y manteniendo solo las sumergidas. Las formas flotantes libres sólo se encuentran en pequeñas lagunas protegidas, ubicadas en las islas del Pacífico, donde no se presentan heladas.

Los cuerpos acuáticos de la Región de Aisén van desde ambientes con corriente (lóticos), agrupados en 7 tipos (ríos, arroyos, arroyos de turberas, saltos de agua, arroyuelos, arroyuelos de bosque $y$ vertientes), a ambientes acuáticos sin corriente (lénticos), representado por 6 situaciones distintas (lagos, lagunas de tierras bajas, lagunas de turberas esfagnosas, lagunas de altura, charcos y marismas) (Tabla 3). Los cuerpos acuáticos con corriente presentaron una mayor cantidad de especies (129) de la flora macrofítica hidrófila, mientras que aquellos lénticos sin corriente, sólo presentaron en conjunto 99 especies (anexo 2). El número de especies acuáticas contabilizadas es superior al número de macrófitos prospectados, debido a que muchas especies se presentan en varios cuerpos acuáticos. No obstante, el promedio de especies por cuerpo

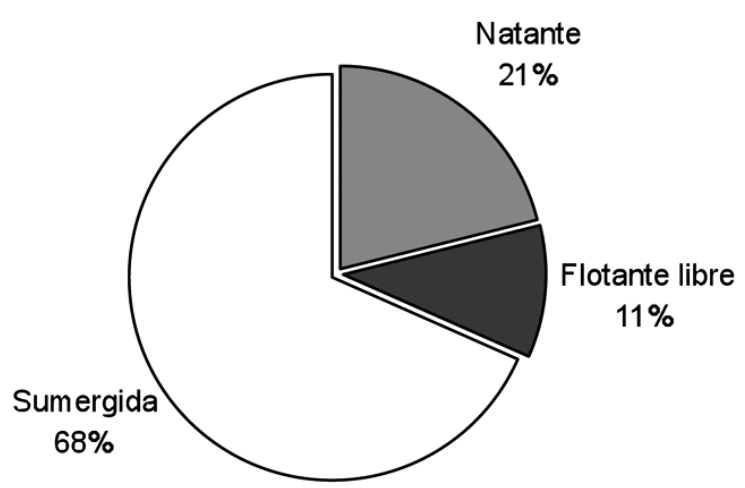

Fig. 4. Distribución de las especies de hidrófitas en tres subtipos: sumergidas, natantes y flotantes libres. 
Tabla 3. Número de especies en los diferentes tipos de cuerpos acuáticos de la región de Aisén. Abreviaturas entre paréntesis.

\begin{tabular}{llr}
\hline \multicolumn{1}{c}{ Cuerpo acuático } & \multicolumn{1}{c}{ Característica } & Especies \\
\hline Ríos (R) & Con mucha corriente & 30 \\
Arroyos (A) & De menor tamaño & 22 \\
Arroyos de turbera (At) & Cruzan o salen de una turbera & 6 \\
Saltos de agua (S) & En cualquier situación & 9 \\
Arroyuelos (Ar) & En cualquiera situación & 37 \\
Arroyuelos de bosques (Ab) & En bosques \\
Vertientes (V) & Taludes de camino \\
Lagos (L) & Lénticos con termoclina \\
Lagunas de tierras bajas (Lb) & Cuerpos lénticos pequeños \\
Lagunas de turberas esfagnosas (Lt) & Cuerpos lénticos turbosos \\
Lagunas de altura (La) & Cuerpos lénticos de altura \\
Charcos (Ch) & Estacionales \\
Marismas salobres (M) & En el litoral pacífico \\
\hline
\end{tabular}

de agua no presenta grandes diferencias variando de 16,5 en aquellos ambientes sin corriente, a 18,4 en lugares con corriente. En todo caso, esta diferencia está dada por los helófitos ribereños que abundan en los ambientes lóticos, los hidrófitos propiamente tal están representados en ellos por solo 9 especies.

La mayor cantidad de especies (44) la presentaron las lagunas de tierras bajas de los ambientes lénticos. Los menores valores correspondieron a 2
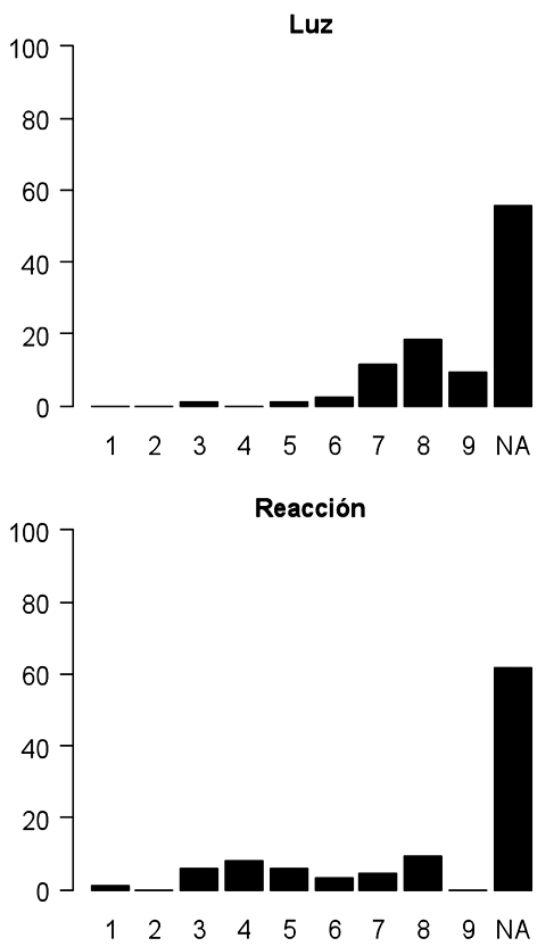

Fig. 5. Espectros de valores indicadores de la flora de los humedales de Aisén. NA indica valores ausentes.
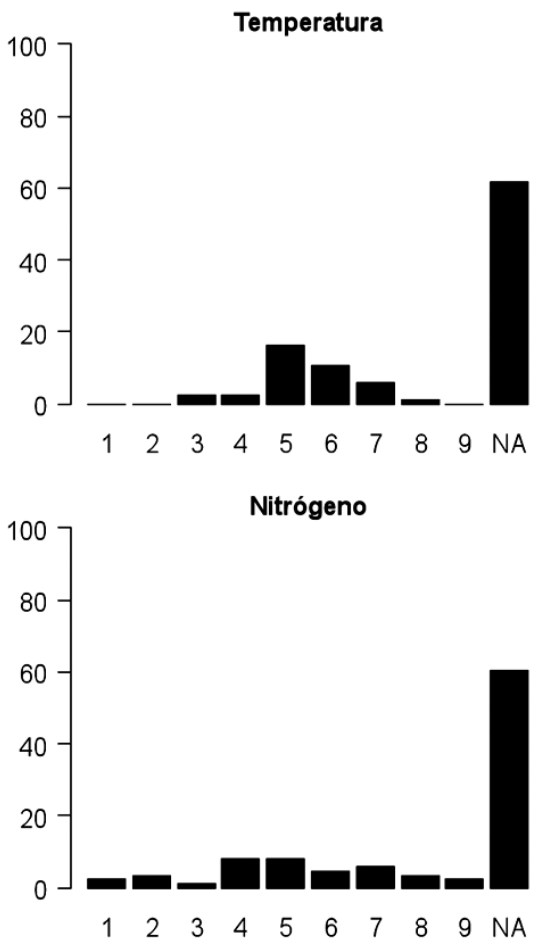

especies por cuerpo de agua, que se presentaron tanto en ambientes lóticos (arroyuelos de bosque) como lénticos (lagunas de altura). En ambos casos hay limitaciones para el crecimiento de los macrófitos, en el primero por falta de luz y en el segundo, por bajas temperaturas.

Varias especies, especialmente de helófitos se presentaron en más de un cuerpo acuático. Las más frecuentes fueron Aster vahlii y Polypogon australis 
que aparecen en 9 de los 13 ambientes acuáticos estudiados. Juncus microcephalus aparece en 8 de ellos. Holcus lanatus, Hydrocotyle chamaemorus, Juncus procerus, Rumex conglomeratus y Scirpus californicus se presentaron en 7 de los cuerpos acuáticos investigados. Por último, Juncus pallescens, Ranunculus repens y Veronica anagallis-aquatica colonizan 6 de los 13 ambientes acuáticos diferenciados. La última especie se presenta también como hidrófito, especialmente en ambientes lóticos.

Tomando en cuenta la distribución de los indicadores ecológicos disponibles sólo para los factores de luz y temperatura se observa una clara tendencia unimodal (Figs. 5 y 6), dominando en el factor luz el valor 8 (luminosidad media a alta) y en el factor temperatura, el valor 5 (clima moderado con oscilaciones térmicas y presencia de heladas). En el resto de los casos la frecuencia de los valores es relativamente homogénea e incluso para la humedad edáfica (Fig. 6) se observa una tendencia bimodal con mayores dominancias en los extremos del espectro. Cabe destacar que para todos los factores, más del $50 \%$ de las especies no contienen datos, lo cual puede estar explicando las tendencias poco claras de los espectros.

Las especies acuáticas son más específicas al seleccionar su ambiente. La forma de vida sumergida que tiene mayor número de especies, crece mayoritariamente en ambientes lénticos sin corriente (14 especies) encontrándose sólo 5 de ellas en los cuerpos de agua lóticos. La forma de vida natante, que en total reunió 4 especies (Agrostis stolonifera, Callitriche palustris, Glyceria multiflora y Potamogeton stenostachys) no tiene grandes diferencias en cuanto al tipo de cuerpo de agua en el cual se desarrolla, presentándo valores relativamente similares en ambos casos (Fig. 4). Finalmente, la forma flotante, la menos frecuente, tiene sus únicos 2 representantes (Azolla filiculoides y Lemna minima) en el ambiente léntico, no presentándose en condiciones lóticas. Su condición de flotantes libres les impide vivir y perdurar en lugares con corriente.

La mayoría (12 de 13) de los cuerpos acuáticos estudiados en este trabajo corresponden a ambientes límnicos, en los cuales fueron prospectadas 71 $(82,56 \%)$ especies de plantas hidrófilas (Tabla 3). El único ambiente salobre encontrado correspondió a marismas que se presentan en pequeños rodales en las escasas playas y riberas bajas de las islas del

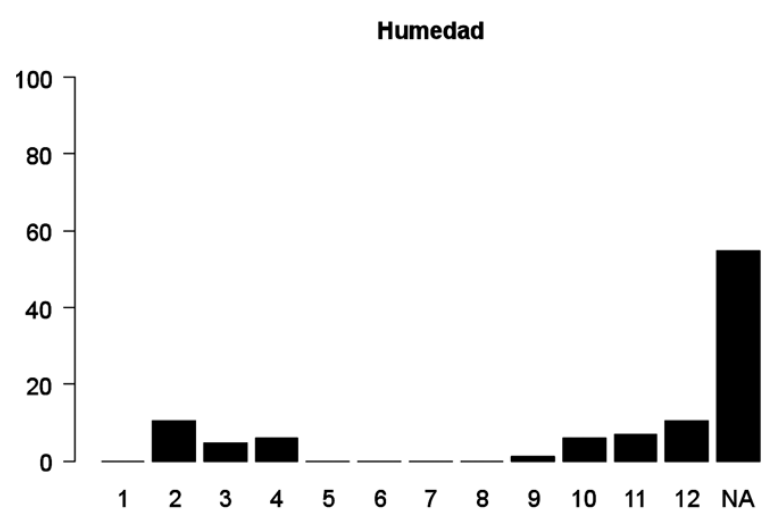

Fig. 6. Espectros de valores indicadores para humedad de la flora de los humedales de Aisén. NA indica valores ausentes.

litoral pacífico. Solo se encontraron 5 especies creciendo en dichos ambientes (Cotula coronopifolia, Puccinellia glaucescens, Triglochin concinna, Ruppia filifolia y Zannichellia palustris), las 3 primeras con características de helófitos emergidos y las 2 últimas, como plantas acuáticas sumergidas. Todas estas especies tienen un comportamiento de halófitos, es decir, son plantas que soportan salinidad. En un lugar intermedio se encuentran las especies capaces de desarrollarse en ambos hábitat (dulciacuícola y salobre), con 10 especies y un 11,63\%.

En el diagrama de ordenación se puede ver la distribución de los tipos de humedales según su composición florística con una clara tendencia de los cuerpos lóticos hacia el lado izquierdo del diagrama, mientras que los cuerpos lénticos lo hacen hacia el derecho (Fig. 7). Cerca de este mismo grupo tienden a conglomerarse los saltos de agua, las vertientes y los arroyuelos, mientras que el resto de los humedales adopta posisiones extremas en el diagrama de ordenación. La agrupación de los arroyos de turbera, las lagunas de turberas esfagnosas y las lagunas de altura en la parte superior del diagrama junto con la ubicación de los charcos en la parte inferior puede estar indicando un gradiente de temperatura y/o contenido de nutrientes en el suelo desde arriba (bajas temperaturas y bajos contenidos de nutrientes) hacia abajo (mayores temperaturas y contenidos de nutrientes).

La historia de las prospecciones sistemáticas de la flora de la Región de Aisén es reciente y relativamente corta, ya que se inició en el año 1935 con Espinosa y hasta la fecha se han podido reunir $13 \mathrm{de}$ 


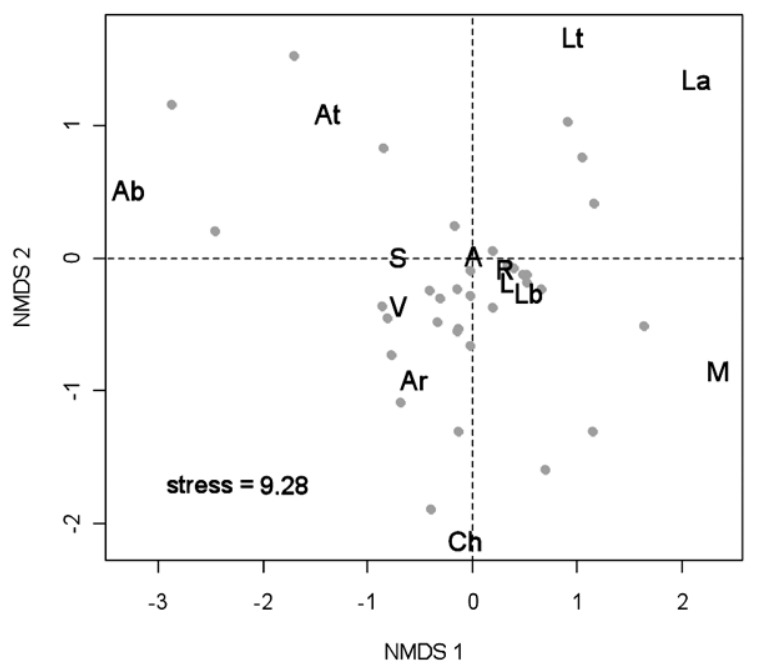

Fig. 7. Distribución de los tipos de hábitats en el nMDS. Puntos grises marcan los centroides de las especies. R: ríos, A: arroyos, At: arroyos de turbera, S: saltos, Ar: arroyuelos, Ab: arroyuelos de bosques, $\mathrm{V}$ : vertientes, $\mathrm{L}$ : lagos, Lb: lagunas de tierras bajas, Lt: lagunas de turberas esfagnosas, La: lagunas de altura, Ch: charcos, M: marismas salobres.

ellas, excluyendo este trabajo (Tabla 4). De este total 9 se refieren especificamente a algún lugar o a toda la Región de Aisén, 3 a la Región de Magallanes y uno, a la distribución de plantas acuáticas en todo el país. Nueve fueron escritos en el siglo pasado y 4 en lo que va corrido del presente. Moore (1982) cita el mayor número de especies acuáticas en sentido amplio (45), le siguen Teiller \& Marticorena (2002) con 42, Álvarez et al. (2010) con 40 y Rodríguez et al. (2008) que citan 38 macrófitos. La mayor cantidad de citas las tiene Gunnera magellanica, especie frecuente en toda la región. Aster vahlii, Marsippospermum grandiflorum, Senecio smithii y Valeriana lapathifolia han sido citadas 9 veces y Myriophyllum quitense, 8. La única especie no mencionada por los autores revisados es Veronica anagallis-aquatica, maleza muy frecuente en la región que, seguramente corresponde a una neófito de reciente introducción y rápida expansión, que eventualmente podría transformarse en invasora. Un caso parecido presenta el helófito Lycopus europaeus, especie que desde su aparición en la Región de Los Ríos con posterioridad a los sismo de mayo de 1960, ha extendido su área de distribución hacia el Sur por el litoral

\section{DISCUSION}

La baja riqueza específica de la flora macrofítica de Aisén corresponde a los patrones normales de distribución latitudinal descritos para plantas acuáticas por Chambers et al. (2008). Comparando la flora acuática de Aisén con la citada en otras localidades de Chile Central (lago Budi, lago Villarrica, lago Vichuquén, laguna de Torca, laguna el Peral y Santuario de la Naturaleza del río Cruces, por ejemplo) la de Aisén aparece como bastante pobre, lo que contrasta con la gran diversidad de ambientes límnicos allí existentes (Hauenstein et al. 1992, Hauenstein et al. 1996, Ramírez et al. 2004, Ramírez \& San

Tabla 4. Número de macrófitos reportados para la zona de estudio a lo largo del tiempo.

\begin{tabular}{llcc}
\hline & & \multicolumn{2}{c}{ Número de especies } \\
Trabajo & Lugar & por trabajo & acumulada \\
Espinosa (1935) & Aysén & 9 & 9 \\
Hambleton (1936) & Canal y Río Baker & 14 & 20 \\
Burkart (1945) & Aysén & 8 & 24 \\
Gunckel (1970) & Patagonia occidental chilena & 16 & 35 \\
Pisano (1976) & Magallanes & 35 & 55 \\
Moore (1982) & Magallanes & 46 & 63 \\
Ramírez et al. (1986) & Chile & 13 & 64 \\
Pisano (1988) & Laguna San Rafael & 22 & 66 \\
Hildebrand-Vogel (1988) & Patagonia occidental & 12 & 69 \\
Teiller \& Marticorena (2002) & Laguna San Rafael & 42 & 73 \\
Vidal (2007) & Torres del Paine & 17 & 73 \\
Rodríguez et al. (2008) & Cuencas ríos Baker y Pascua & 38 & 77 \\
Alvarez et al. (2010) & Archipiélago de Los Chonos & 40 & 85 \\
Este trabajo & Aisén & 86 & 86
\end{tabular}


Martín 1984, Ramírez et al. 1987, Ramírez et al. 1991). Las causas de esta escasa representación hay que buscarlas primero en las diferencias climáticas (mucho más frío en Aisén) pero sobre todo, en las características de los cuerpos de agua de esa región que son extremadamente oligotróficos debido a su origen glaciar (De los Ríos \& Soto 2009). Pero también influye la escasa alteración humana de dichos cuerpos que ha permitido la mantención de esa oligotrofía, como lo señala la presencia de Isoetes savatierii, Marsippospermum grandiflorum, Potamogeton stenostachys y Tetroncion magellanicum, indicadores de condiciones prístinas (San Martín et al. 2003). En Chile central y, especialmente, en los humedales de la cordillera costera, la eutroficación es un problema muy grande que facilita el desarrollo exagerado de la flora acuática, especialmente sumergida y natante, lo que dificulta su uso (Ramírez et al. 2004). En la Región de Aisén en cambio, pocas especies de plantas acuáticas son abundantes, formando grandes poblaciones. Ellas aseguran la mantención de la transparencia del agua impidiendo el paso a un estado turbio en el cual dominen especies fitoplanctónicas (Scheffer et al. 1993).

La proporción de especies por grupos vegetales, es la normal para los cuerpos acuáticos chilenos, aunque la diversidad de familias y géneros es un poco menor. No obstante, revisiones sistemáticas de grupos como Ranunculus (especialmente el complejo $R$. aquatilis que tiene poca afinidad con las especies europeas, como se las considera actualmente) podrían incrementar la diversidad de especies.

El carácter azonal de la flora acuática no permite determinar con exactitud el origen fitogeográfico de las especies, también influyen los problemas sistemáticos existentes, como Ranunculus aquatilis, que podría ser una especie nativa. De la misma manera se considera que Potamogeton natans (especie introducida) en la Región de Aisén, corresponde a Potamogeton stenostachys (especie nativa). En todo caso el predominio de especies nativas confirma la poca intervención humana de los hábitats. Sin embargo, últimamente han comenzado a aflorar neófitos (Lycopus europaeus y Verónica anagallis-aquatica) lo que señala una incipiente intervención humana, que habría que considerar y tratar de controlar. Un caso interesante lo constituye la especie europea Hippuris vulgaris, especie muy abundante en la Patagonia de la cual se desconoce la época y forma de arribo a Chile y cuya dispersión se detuvo en la Patagonia chilena, sin avanzar hacia el centro el país (Pisano 1976).

El espectro biológico de Raunkiaer corresponde a lo indicado en la literatura para esas latitudes, como lo demuestra la escasez de planta anuales indicadoras de sequía, que sólo están presentes en las riberas más secas de los ríos y lagos. La proporción entre plantas palustres y plantas acuáticas corresponde a la proporción normal, ya que como se dijo solo indica la abundancia de hábitats palustres en la zonación ribereña. La ausencia de grandes macrófitos flotantes libres está de acuerdo con la abundancia de ambientes de fuerte corriente y con el clima más frío (Meerhoff \& Mazzeo 2004, Hajek \& Di Castri 1975).

Es difícil separar con exactitud las especies de flora acuática perteneciente a los diferentes ambientes con y sin corriente ya que en los ríos prospectados la mayor cantidad y diversidad de macrófitos se encuentra en bañados, remansos o "aguas muertas" que precisamente tienen características de lagunas lénticas que se forman en el curso inferior (potamon), por lo demás, a menudo el gran caudal del curso intermedio (rithron) de los ríos, no permite la colonización de las riberas (Ramírez \& San Martín 2006b). Esta tendencia también se puede observar en el diagrama de ordenación mostrado en la Fig. 7. Los lagos y las lagunas de tierras bajas están bastante cerca de los arroyos y ríos, lo que indica una alta similitud florística entre ellos.

La flora de arroyuelos y lagunas de altura está integrada solo por especies nativas, debido al carácter extremo de los biótopos, en los primeros son importantes Ourisia coccinea y O. ruelloides. Los ambientes acuáticos de tierras bajas son los más favorables para el desarrollo de la flora estudiada. Las vertientes de tierras bajas, muchas de ellas en taludes de camino, tienen una flora muy especial dominada por la robusta hierba Gunnera tinctoria, acompañada de Valeriana lapathifolia y V. carnosa. Aunque no fueron prospectadas en la región de Aisén existen lagunas efímeras estacionales, que podrían contribuir con una mayor cantidad de especies a la flora hidrófila, especialmente con plantas anfibias, sin embargo estas lagunas exigen estacionalidad en el muestreo, lo cual no siempre es posible. Los 
macrófitos natantes presentan grandes tamaños $y$, además, colonizan ambientes lóticos de mucha corriente.

Los cuerpos de agua se clasifican según su nivel de salinidad en salinos (marinos), dulces (límnicos) y salobres (estuarinos). En los primeros y en las regiones de clima templado, no existen plantas acuáticas y la vegetación ribereña está formada exclusivamente por algas y los últimos son ocupados por marismas, que contienen halófitos muy especializados. Hasta el momento se desconocía la existencia de marismas en las islas del Archipiélago de Los Chonos, seguramente ellas eran más abundantes en el pasado, ya que con los sismos de mayor de 1960, experimentaron un alzamiento del terreno, dejando secos muchos ambientes costeros (Plafker 1972, Fuenzalida 1978). El pequeño tamaño de los rodales existentes actualmente, está indicando una recuperación de ellas. Cabe mencionar que, al igual a lo reportado por Eskuche (2005) para humedales litorales en los canales magallánicos más al sur, las altas precipitaciones reducen el efecto de la salinidad, por lo cual también se podría observar una flora no especializada en las marismas de esta región. Los resultados de los modelos de regresión se muestran en la Tabla 5. Siguiendo el valor de AIC son las plantas de aguas salobres las que están más fuertemente correlacionadas con la ordenación (AIC $=18,37$ ), ello debido a que estas plantas sólo están presentes en las marismas, por ello mismo el efecto del eje $1\left(e^{\beta 1}\right)$ es bastante alto, pues las marismas se encuentran al extremo derecho de la ordenación.
Similar es el caso de las hidrófitas flotantes (AIC $=28,27$ ), las cuales están representadas por sólo dos especies (Azolla filiculoides y Lemna minima), ambas presentes en las lagunas bajas. A estos grupos de características les sigue el de las terófitas (AIC = 37,37), representado por sólo tres especies (Juncus bufonius, Montia fontana y Ranunculus uniflorus) que aparecen en los charcos, en las marismas y en las lagunas bajas.

Los resultados de la prospección histórica deben ser evaluados de acuerdo con la naturaleza de los trabajos florísticos revisados. Especialmente cuando se trata de los trabajos de Moore (1982) y Vidal (2007), las floras de Tierra del Fuego y del Parque Nacional Torres del Paine, respectivamente. Con excepción del trabajo de Pisano (1976) la mayoría de los existentes se refiere específicamente a la flora terrestre, por lo que no hay un especial énfasis en el grupo de plantas acuáticas. No obstante, y a pesar de eso, el presente trabajo sólo contribuyó con una especie no citada Verónica anagallis-aquatica, que seguramente es un neófito. A ella podría sumarse Lycopus europaeus también con carácter de neófito, aunque aún sin características de invasor. Dado que el carácter de especies introducidas presenta poca correlación con la diversidad florística según los modelos de regresión logística (Tabla 5), la distribución de neófitas es relativamente homogénea en todos los tipos de ambientes acuáticos. Sin embargo, es necesario hacer revisiones más exhaustivas de los trabajos existentes, ya que en ellos hay valiosa información dispersa, que debe ser actualizada sistemática

Tabla 5. Parámetros de los modelos de correlación logística entre las características específicas y la distribución de la vegetación en el nMDS. $\beta 0$ es el intercepto de la función linearizada, $e^{\beta 1}$ y $e^{\beta 2}$ son los efectos de la primera y segunda dimensión del diagrama de ordenación, respectivamente, y AIC es el criterio de Akaike.

\begin{tabular}{lcccc}
\hline Factor & $\beta 0$ & $e^{\beta 1}$ (nMDS1) & $e^{\beta 2}$ (nMDS2) & AIC \\
\hline De agua salobre & $-35,10$ & $227.761,45$ & 0,00 & 18,37 \\
Hidrófitas flotantes & $-4,90$ & 1,92 & 1,06 & 28,27 \\
Terófitas & $-6,27$ & 2,70 & 0,08 & 37,37 \\
Caméfitas & $-3,56$ & 0,47 & 0,73 & 72,02 \\
Hidrófitas natantes & $-2,87$ & 1,91 & 2,27 & 96,98 \\
Hidrófitas submersas & $-2,98$ & 2,17 & 0,53 & 117,97 \\
Helófitas & 2,01 & 0,43 & 0,96 & 178,12 \\
De agua dulce & 1,85 & 0,33 & 1,12 & 195,61 \\
Ambos tipos de salinidad & $-1,75$ & 1,66 & 1,22 & 195,97 \\
Introducidas & $-1,63$ & 1,22 & 0,71 & 221,74 \\
Nativas & 1,63 & 0,82 & 1,41 & 221,74 \\
Criptófitas & $-1,10$ & 1,79 & 1,79 & 243,31 \\
Hemicriptófitas & 0,87 & 0,63 & 0,86 & 277,30 \\
\hline
\end{tabular}


Tabla 6. Aparición de especies neófitas en los registros por décadas. Década 00' contiene todos los registros a la fecha.

\begin{tabular}{lccl}
\hline \multirow{2}{*}{ Década } & \multicolumn{2}{c}{ Especies acumuladas } & Nuevos registros \\
\cline { 2 - 3 } $30^{\prime}$ & total & introducidas & \\
$70^{\prime}$ & 20 & 2 & Juncus bufonius, Rumex conglomeratus \\
$80^{\prime}$ & 55 & 5 & Carex pseudocyperus, Hippuris vulgaris, Ranunculus repens \\
$00^{\prime}$ & 69 & 8 & Agrostis stolonifera, Callitriche palustris, Holcus lanatus \\
& 86 & 13 & Cotula coronopifolia, Lycopus europaeus, Lythrum portula, Potentilla \\
\hline
\end{tabular}

y nomenclaturalmente. Todo esto permitirá conocer, manejar y conservar la biodiversidad de la región.

Un detalle del incremento en registros de plantas introducidas (neófitas) se muestra en la Tabla 6. Aquí cabe mencionar que la aparición de las especies en los registros no se asocia necesariamente a su introducción. La proporción de neófitos en los registros estudiados ha ido en incremento, de un 9 a $10 \%$ entre las décadas de los 1930' y 1970', hasta un $15 \%$ en los registros actuales. Dicha tendencia no está libre de sesgos en los muestreos, sin embargo representa un tema interesante de estudiar más a fondo.

\section{LITERATURA CITADA}

Álvarez, M., C. San Martín, C. Novoa, G. Toledo \& C. Ramírez 2010. Diversidad florística, vegetacional y de hábitats en el Archipiélago de Los Chonos (Región de Aisén, Chile). Anales Instituto Patagonia (Chile) 38(1):34-55

Arber, A. 1963. Water plants: a study of aquatic angiosperms. University Press, Cambridge $436 \mathrm{pp}$.

Burkart, A. 1945. Enumeración sistemática de las plantas coleccionadas durante la expedición patagónica en el territorio de Aisén, Chile en Enero y Febrero de 1939 por la señora Ilse Von Rentzell. Darwiniana 7:153-170

Campos, H. 1979. El recurso de agua dulce en Chile. Boletín Informativo Limnológico 3:6-17

Chambers, P.A., P. Lacoul, K.J. Murphy \& S.M. Thomaz 2008. Global diversity of aquatic macrophytes in freshwater. Hydrobiologia 595:9-26

Cook, C., B. Gut, E.M. Rix, E. Schneller \& M. Seita 1974. Water plants of the world $-a$ manual for the identification of the genera of freshwater macrophytes. Dr. W. Junk, La Haya 425 pp.
Davis, J., D. Blasco \& M. Carbonell 1996. Manual de la Convención Ramsar - una guía a la convención sobre los humedales de importancia internacional. Gland $211 \mathrm{pp}$.

De los Ríos, P. \& D. Soto 2009. Estudios limnológicos en lagos y lagunas del Parque Nacional Torres del Paine (51으, Chile). Anales Instituto Patagonia (Chile) 47(1):63-71

Ellenberg, H. 1979. Zeigerwerte der Gefässpflanzen Mitteleuropas. Scripta Geobotanica 9:5-97

Eskuche, U. 2005. Las comunidades vegetales litorales de los lagos patagónicos y de los canales magallánicos. Folia Botanica et Geobotanica Correntesiana 18:1-31

Espinosa, M. 1935. Plantas de Aisén. Boletín del Museo Nacional de Historia Natural 14:65-82

Fernández, A., A. Rivera \& C.R. Ramírez 2006. Variaciones recientes de glaciares entre $41^{\circ}$ $\mathrm{S}$ y $49^{\circ} \mathrm{S}$ y su relación con los cambios climáticos. Revista Geográfica 139:39-69

Frey, W. \& R. Lösch 1998. Lehrbuch der Geobotanik - Pflanze und Vegetation in Raum und Zeit. Gustav Fischer, Stuttgart 436 pp.

Fuenzalida, R. 1978. Nuevos antecedentes de cambios de morfología costera después de los sismos de 1960, Archipiélago de Los Chonos. Trapananda 1:71-75

Gunckel, H. 1970. Algunas especies vegetales de la Patagonia occidental chilena coleccionadas por la Segunda Expedición Japonesa. Boletín Universidad de Chile 106:17-28

Hajek, E.R. \& F. Di Castri. 1975. Bioclimatografía de Chile. Universidad Católica de Chile, Santiago 144 pp.

Hambleton, H. 1936. La vegetación del canal y río Baker (Patagonia occidental). Revista Argentina de Agronomía 3:159-173

Hauenstein, E., C. Ramírez, M. González \& C. San Martín 1992. Comparación de la flora macrofítica de tres lagos del Centro-Sur de 
Chile (Budi, Llanquihue y Cayutue). Revista Geográfica de Valparaíso 22-23:175-193

Hauenstein, E., C. Ramírez, M. González, L. Leiva \& C. San Martín 1996. Flora hidrófila del Lago Villarrica (IX Región, Chile) y su importancia como elemento indicador de contaminación. Medio Ambiente 13:88-96

Hildebrandt-Vogel, R. 1988. Ersatzgesellschaften des nordwestpatagonischen Lobeerwaldes in Chile zwischen 42 uns $45^{\circ}$ s. Br. Flora 180:161-176

Hutchinson, G.E. 1975. A treatise on Limnology. Vol. 3, Limnological botany. New York 660 pp.

Lepš, J. \& P. Šmilauer 2003. Multivariate analysis of ecological data using CANOCO. Cambridge University $269 \mathrm{pp}$.

Leyer, I. \&. K. Wesche 2007. Multivariate Statistik in der Ökologie. Springer, Berlin 221 pp.

Mardones, M., J. Jara \& J. Vargas 2007. El patrón hidrográfico de la cuenca del río Blanco: control tectónico y geomorfológico. Revista de Geografía Norte Grande 38:79-98

Marticorena, C. \& M. Quezada 1985. Catálogo de la flora vascular de Chile. Gayana Botanica 42:5-157

Matthei, O. 1995. Manual de las malezas que crecen en Chile. Alfabeta, Concepción 545 pp.

Meerhof, S.W. \& N. Mazzeo 2004. Importancia de las plantas flotantes libres de gran porte en la conservación y rehabilitación de lagos someros de Sudamérica. Ecosistemas 13:13-22

Menard, S.W. 2002. Applied logistic regression analysis. $2^{a}$ ed., Sage, California. Quantitative Applications in the Social Sciences 106:128 pp.

Moore, D. 1982. Flora of Tierra del Fuego. Anthony Nelson, London 396 pp.

Mueller-Dombois, D. \& H. Ellenberg 1974. Aims and Methods of Vegetation Ecology. John Wiley, New York 547 pp.

Murúa, R., J.C. Miranda \& C. Ramírez 1993. Necesidad de una política de "bosques para leña". Ambiente y Desarrollo 9:75-80

Plafker, G. 1972. The Alaska earthquake of 1964 and Chilean earthquake of 1960: implications for arc tectonics. Journal of Geophysical Research 77:901-925

Pisano, E. 1988. Sectorización fitogeográfica del archipiélago sud patagónico-fueguino: II. Vegetación y flora vascular del área del Par- que Nacional "Laguna San Rafael", Aisén. Anales Instituto Patagonia, Serie Cs. Nat. (Chile) 18:5-34

Pisano, E. 1976. Cormófitos acuáticos de Magallanes. Anales Instituto Patagonia (Chile) 7:115-136

Ramírez, C. \& C. San Martín 2006a. Diversidad de macrófitos chilenos. En: Vila, I., A. Veloso, R. Schlatter \& C. Ramírez (eds.), Macrófitas y vertebrados de los sistemas límnicos de Chile. pp. 21-61 Editorial Universitaria, Santiago

Ramírez, C. \& C. San Martín 2006b. Ecosistemas dulceacuícolas. En: Saball, P., M.T.K. Arroyo, J.C. Castilla, C. Estades, J.M. Ladrón de Guevara, S. Larraín, C. Moreno, F. Rivas, J. Rovira, A. Sánchez \& L. Sierralta (eds.), Biodiversidad de Chile. Patrimonio y Desafíos. pp. 112-124 Comisión Nacional del Medio Ambiente, Santiago

Ramírez, C. \& C. San Martín 2006c. Flora acuática. En: Saball, P., M Arroyo, J.C. Castilla, C. Estades, J.M. Ladrón de Guevara, S. Larraín, C. Moreno, F. Rivas, J. Rovira, A. Sánchez \& L. Sierralta (eds.). Biodiversidad de Chile. Patrimonio y Desafíos. pp. 364-369 Comisión Nacional del Medio Ambiente, Santiago

Ramírez, C. \& J. San Martín 1984. Hydrophylous vegetation of a coastal lagoon in Central Chile. Ecological Environmental Science 10:93-100 Ramírez, C. \& E. Stegmaier 1982. Formas de vida en hidrófitos chilenos. Medio Ambiente 6:43-54

Ramírez, C., D. Contreras \& J. San Martín 1986. Distribución geográfica y formas de vida en hidrófitos chilenos. Actas VIII Congreso Nacional de Geografía, Publicación Especial del Instituto Geográfico Militar de Chile 1:103-110

Ramírez, C., R. Mac Donald \& C. San Martín 1996. Riesgos ambientales de la transformación de suelos en la Región de Los Lagos. Ambiente y Desarrollo 12:82-88

Ramírez, C., C. San Martín \& H. Rubilar 2002. Una propuesta para la clasificación de los humedales chilenos. Revista Geográfica de Valparaíso 32-33:265-273

Ramírez, C., C. San Martín \& J. San Martín 2004. Colmatación por macrófitos del complejo lacustre Vichuquén (VII Región, Chile) y clave de determinación. Revista Geográfica de Chile Terra Australis 49:179-196 
Ramírez, C., R. Godoy, D. Contreras \& E. Stegmaier 1982. Guía de plantas acuáticas y palustres Valdivianas. Instituto de Botánica, Universidad Austral de Chile, Valdivia 52 pp.

Ramírez, C., C. San Martín, D. Contreras \& J. San Martín 1989. Flora de las marismas del centrosur de Chile. Medio Ambiente 10:11-24.

Ramírez, C., C. San Martín, R. Medina \& D. Contreras 1991. Estudio de la flora hidrófila del Santuario de la Naturaleza "Río Cruces" (Valdivia, Chile). Gayana Botánica 48:67-80

Ramírez, C., J. San Martín, C. San Martín \& D. Contreras 1987. Estudio florístico y vegetacional de la laguna El Peral, Quinta Región de Chile. Revista Geográfica de Valparaíso 18:105-120

Rodríguez, R. 1995. Pteridophyta. En: Marticorena C. \& R. Rodríguez (eds.). Flora de Chile, Pteridophyta - Gymnospermae. pp. 1:119-309 Universidad de Concepción, Chile

Rodríguez, R. \& V. Dellarossa 1998. Plantas vasculares acuáticas en la Región del Bio-Bío. Universidad de Concepción 38 pp.

Rodríguez, R., A. Marticorena \& E. Teneb 2008. Plantas vasculares de los ríos Baker y Pascua, Región de Aisén, Chile. Gayana Botanica 65:39-70

Rojas, P. \& J. Leidich 2006. Turismo de naturaleza, desarrollo local sustentable y megaproyectos hidroeléctricos en la Patagonia Chilena. Sociedad Hoy 11:87-108

Rojas, P. \& J. Leidich 2009. Las crecidas del río Baker, Provincia de Capitán Prat, XI región de Aisén, Chile. (http://www.ecosistemas. cl/1776/articles-85339_recurso_1.pdf)
Rovere, A., A. Premoli \& A. Newton 2002. Estado de conservación del ciprés de las Guaitecas (Pilgerodendron uviferum (Don) Florín) en Argentina. Bosque 23:11-19

San Martín, C. C. Ramírez \& M. Álvarez 2003. Macrófitos como bioindicadores: Una propuesta metodológica para caracterizar ambientes dulciacuícolas. Revista Geográfica de Valparaíso 34:243-253

San Martín, C., J. Villagra \& C. Novoa 2009. Comparación de manejos pratenses del centro-sur de Chile utilizando valores bioindicadores de Ellenberg. Gayana Botánica 66(2):158-170

Scheffer, M., S.H., Hosper, M.L. Meijer, B. Moss \& E. Jeppesen 1993. Alternative equilibria in shallow lakes. Trends in Ecology and Evolution 8:275-279

Teiller, S. \& C. Marticorena 2002. Riqueza florística del Parque Nacional Laguna San Rafael, XI Región, Chile. Boletín del Museo Nacional de Historia Natural 51:43-73

Vidal, O. 2007. Flora Torres del Paine, Guía de Campo. Fantástico Sur Birding, Punta Arenas 345 pp.

Vidal OJ., JR. Bannister, V. Sandoval, Y. Pérez \& C. Ramírez 2011. Woodland communities in the Chilean cold-temperate zona (Baker and Pascua basins): Floristic composition and morphoecological transition. Gayana Botánica: en prensa.

Villagra, J., D. Montenegro, C. San Martín, C. Ramírez \& I. Álvarez 2009. Estudio de la flora liquénica de las turberas de la Comuna de Tortel (Región de Aisén), Patagonia chilena. Anales Instituto Patagonia (Chile) 37:53-62 
Anexo 1. Especies, Familia, Nombre común Origen fitogeográfico $(\mathrm{O})$, Hábitat $(\mathrm{H})$. Forma de vida $(\mathrm{FV})$ y Salinidad del agua (Ha) de la flora acuática de Aysén.

\begin{tabular}{|c|c|c|c|c|c|c|}
\hline Especie & Familia & Nombre común & $\mathrm{O}$ & $\mathrm{H}$ & $\mathrm{FV}$ & $\mathrm{Ha}$ \\
\hline Agrostis stolonifera L. & Poaceae & Chépica rastrera & I & $\mathrm{Hi}$ & $\mathrm{Cr}$ & $\mathrm{D}$ \\
\hline Anagallis alternifolia Cav. & Primulaceae & Pimpinela & $\mathrm{N}$ & $\mathrm{He}$ & $\mathrm{H}$ & $\mathrm{D}, \mathrm{S}$ \\
\hline Aster vahlii (Gaud.) H. et A. & Asteraceae & Margarita del pantano & $\mathrm{N}$ & $\mathrm{He}$ & $\mathrm{Cr}$ & $\mathrm{D}, \mathrm{S}$ \\
\hline Azolla filiculoides Lam. & Azollaceae & Flor del pato & $\mathrm{N}$ & $\mathrm{Hi}$ & $\mathrm{Cr}$ & $\mathrm{D}$ \\
\hline Baccharis sagittalis (Less.) DC. & Asteraceae & Verbena de tres esquinas & $\mathrm{N}$ & $\mathrm{He}$ & $\mathrm{C}$ & $\mathrm{D}, \mathrm{S}$ \\
\hline Blechnum chilense (Kaulf.) Mett. & Blechnaceae & Quil-Quil & $\mathrm{N}$ & $\mathrm{He}$ & $\mathrm{H}$ & $\mathrm{D}$ \\
\hline Calceolaria tenella Poep. & Calceolariaceae & Topa-Topa chica & $\mathrm{N}$ & $\mathrm{He}$ & $\mathrm{H}$ & $\mathrm{D}$ \\
\hline Callitriche lechleri (Hegelm.) Fasset & Callitrichaceae & Estrella de agua & $\mathrm{N}$ & $\mathrm{Hi}$ & $\mathrm{Cr}$ & $\mathrm{D}$ \\
\hline Callitriche antarctica Hegelm. ex Hegelm. & & Yerbecilla & $\mathrm{N}$ & $\mathrm{Hi}$ & $\mathrm{Cr}$ & $\mathrm{D}$ \\
\hline Callitriche terrestris Rafin. & & Huenchecó & $\mathrm{N}$ & $\mathrm{Hi}$ & $\mathrm{Cr}$ & $\mathrm{D}$ \\
\hline Callitriche palustris $\mathrm{L}$ & & Estrella de agua & I & $\mathrm{Hi}$ & $\mathrm{Cr}$ & $\mathrm{D}$ \\
\hline Caltha sagittata Cav. & Ranunculaceae & Maicillo & $\mathrm{N}$ & $\mathrm{He}$ & $\mathrm{H}$ & $\mathrm{D}$ \\
\hline Cardamine bonariensis A.L. Juss. ex Pers. & Brassicaceae & Berro blanco & $\mathrm{N}$ & $\mathrm{He}$ & $\mathrm{H}$ & $\mathrm{D}$ \\
\hline Cardamine glacialis (G. Forst.) DC. & & No conocido & $\mathrm{N}$ & $\mathrm{He}$ & $\mathrm{H}$ & $\mathrm{D}$ \\
\hline Carex banksii Boot. & Cyperaceae & Cortadera & $\mathrm{N}$ & $\mathrm{He}$ & $\mathrm{H}$ & $\mathrm{D}$ \\
\hline Carex curta Good. & & Cortadera chica & $\mathrm{N}$ & $\mathrm{He}$ & $\mathrm{H}$ & $\mathrm{D}$ \\
\hline Carex darwinii Boot. & & No conocido & $\mathrm{N}$ & $\mathrm{He}$ & $\mathrm{H}$ & $\mathrm{D}$ \\
\hline Carex macloviana D'Urv. & & No conocido & $\mathrm{N}$ & $\mathrm{He}$ & $\mathrm{H}$ & $\mathrm{D}$ \\
\hline Carex pseudocyperus L. & & Cortadera grande & I & $\mathrm{He}$ & $\mathrm{H}$ & $\mathrm{D}$ \\
\hline Carex riparia Curtis & & Cortadera azul & $\mathrm{N}$ & $\mathrm{He}$ & $\mathrm{H}$ & $\mathrm{D}$ \\
\hline Cotula coronopifolia L. & Asteraceae & Botón de oro africano & I & $\mathrm{He}$ & $\mathrm{H}$ & $\mathrm{S}$ \\
\hline Crassula peduncularis (Schlecht.) Reiche & Crassulaceae & Chacalcura & $\mathrm{N}$ & $\mathrm{Hi}$ & $\mathrm{Cr}$ & $\mathrm{D}$ \\
\hline Cyperus eragrostis Lam. & Cyperaceae & Cortadera & $\mathrm{N}$ & $\mathrm{He}$ & $\mathrm{H}$ & $\mathrm{D}$ \\
\hline Dysopsis glechomoides (A.Rich.) Muell. Arg. & Euphorbiaceae & No conocido & $\mathrm{N}$ & $\mathrm{He}$ & $\mathrm{H}$ & $\mathrm{D}$ \\
\hline Epilobium australe Poepp. et Hausskn. & Onagraceae & Epilobio & $\mathrm{N}$ & $\mathrm{He}$ & $\mathrm{H}$ & $\mathrm{D}$ \\
\hline Epilobium ciliatum Raf. & & Epilobio & $\mathrm{N}$ & $\mathrm{He}$ & $\mathrm{H}$ & $\mathrm{D}$ \\
\hline Eleocharis melanostachys (D’Urv.) C.B. Clar. & Cyperaceae & Quilmén negro & $\mathrm{N}$ & $\mathrm{He}$ & $\mathrm{H}$ & $\mathrm{D}$ \\
\hline Eleocharis pachycarpa Desv. & & Quilmén & $\mathrm{N}$ & $\mathrm{He}$ & $\mathrm{H}$ & $\mathrm{D}$ \\
\hline Equisetum bogotense H.B.K. & Equisetaceae & Limpiaplata & $\mathrm{N}$ & $\mathrm{He}$ & $\mathrm{Cr}$ & $\mathrm{D}$ \\
\hline Geum magellanicum Pers. & Rosaceae & Llallante & $\mathrm{N}$ & $\mathrm{He}$ & $\mathrm{H}$ & $\mathrm{D}$ \\
\hline Glyceria multiflora Steud. & Poaceae & Gliceria & $\mathrm{N}$ & $\mathrm{Hi}$ & $\mathrm{Cr}$ & $\mathrm{D}$ \\
\hline Gunnera magellanica Lam. & Gunneraceae & Dinacho & $\mathrm{N}$ & $\mathrm{He}$ & $\mathrm{H}$ & $\mathrm{D}$ \\
\hline Gunnera tinctoria (Mol.) Mirb. & & Pangue & $\mathrm{N}$ & $\mathrm{He}$ & $\mathrm{H}$ & $\mathrm{D}$ \\
\hline Hippuris vulgaris L. & Hippuridaceae & Pinito de agua & I & $\mathrm{He}$ & $\mathrm{Cr}$ & $\mathrm{D}$ \\
\hline Holcus lanatus L. & Poaceae & Pasto dulce & I & $\mathrm{He}$ & $\mathrm{H}$ & $\mathrm{D}$ \\
\hline Hydrocotyle chamaemorus Cham. et Schlecht. & Hydrocotylaceae & Tembladerilla & $\mathrm{N}$ & $\mathrm{He}$ & $\mathrm{H}$ & $\mathrm{D}$ \\
\hline Isoetes savatieri Franchet & Isoetaceae & Isete & $\mathrm{N}$ & $\mathrm{Hi}$ & $\mathrm{Cr}$ & $\mathrm{D}$ \\
\hline Juncus bufonius L. & Juncaceae & Junquillo anual & I & $\mathrm{He}$ & $\mathrm{T}$ & $\mathrm{D}$ \\
\hline Juncus microcephalus H.B.K. & & Junquillo rojo & $\mathrm{N}$ & $\mathrm{He}$ & $\mathrm{H}$ & $\mathrm{D}$ \\
\hline Juncus pallescens Lam. & & Hierba de la vaca & $\mathrm{N}$ & $\mathrm{He}$ & $\mathrm{H}$ & $\mathrm{D}$ \\
\hline Juncus planifolius R. Br. & & Junquillo & $\mathrm{N}$ & $\mathrm{He}$ & $\mathrm{H}$ & $\mathrm{D}$ \\
\hline Juncus procerus E. Mey. & & Junquillo grande & $\mathrm{N}$ & $\mathrm{He}$ & $\mathrm{H}$ & $\mathrm{D}$ \\
\hline Juncus stipulatus Nees. Et Meyen & & Junquillito & $\mathrm{N}$ & $\mathrm{He}$ & $\mathrm{H}$ & $\mathrm{D}$ \\
\hline Lemna minima Phil. & Lemnaceae & Lenteja de agua & $\mathrm{N}$ & $\mathrm{Hi}$ & $\mathrm{Cr}$ & $\mathrm{D}$ \\
\hline Lilaeopsis macloviana (Gaud.) A.W. Hill. & Apiaceae & No conocido & $\mathrm{N}$ & $\mathrm{Hi}$ & $\mathrm{Cr}$ & $\mathrm{D}$ \\
\hline Lilaea scilloides (Poir.) Haum. & Lilaeaceae & Lilea & $\mathrm{N}$ & $\mathrm{He}$ & $\mathrm{H}$ & $\mathrm{D}$ \\
\hline Limosella australis $\mathrm{R} . \mathrm{Br}$. & Scrophulariaceae & Limosela & $\mathrm{N}$ & $\mathrm{Hi}$ & $\mathrm{Cr}$ & $\mathrm{D}$ \\
\hline Litorella australis Griseb. & Plantaginaceae & Litorela & $\mathrm{N}$ & $\mathrm{He}$ & $\mathrm{H}$ & $\mathrm{D}$ \\
\hline
\end{tabular}


Lobelia alata (Wedd.) Lammers

Lycopus europaeus L.

Lythrum portula L.

Marsippospermum grandiflorum (L.f.) Hook.

Mimulus parviflorus Lindl.

Mimulus glabratus H.B.K.

Mimulus luteus L.

Montia fontana L.

Myriophyllum quitense H.B.K.

Ourisia coccinea (Cav.) Pers.

Ourisia ruelloides (L.f.) Kuntze

Polypogon australis (Cav.) Trin. Ex Steud.

Potamogeton stenostachys K. Schum.

Potentilla anserina L.

Pratia repens Gaud.

Puccinellia glaucescens (Phil.) Parodi

Ranunculus biternatus J.E. Sm.

Ranunculus uniflorus Phil. Ex Reiche

Ranunculus aquatilis L.

Ranunculus cymbalaria Pursch.

Ranunculus minutiflorus Bert. ex Phil.

Ranunculus peduncularis J.E. Sm.

Ranunculus pseudotrullifolius Skottsb.

Ranunculus repens L.

Rumex conglomeratus Murr.

Ruppia filifolia (Phil.) Skottsb.

Scirpus californicus (C.A. Mey.) Steud.

Scirpus cernuus Vahl.

Scirpus inundatus (R. Br.) Poir.

Senecio acanthifolius Hombr. et Jacquinot

Senecio fistulosus Poepp. ex Less.

Senecio smithii DC.

Tetroncium magellanicum Willd.

Triglochin concinna Davy

Triglochin striata R. et P.

Valeriana lapathifolia Vahl.

Veronica anagallis-aquatica L.

Zannichellia palustris L.

\begin{tabular}{|c|c|c|c|c|c|}
\hline Lobeliaceae & No conocido & $\mathrm{N}$ & $\mathrm{He}$ & C & $\mathrm{D}, \mathrm{S}$ \\
\hline Lamiaceae & Pata de lobo & I & $\mathrm{He}$ & $\mathrm{C}$ & $\mathrm{D}$ \\
\hline Lythraceae & Romerillo rojo & I & $\mathrm{Hi}$ & $\mathrm{Cr}$ & $\mathrm{D}$ \\
\hline Juncaceae & Junco canasto & $\mathrm{N}$ & $\mathrm{He}$ & $\mathrm{H}$ & $\mathrm{D}$ \\
\hline \multirow{3}{*}{ Scrophulariaceae } & Berro chico & $\mathrm{N}$ & $\mathrm{He}$ & $\mathrm{C}$ & $\mathrm{D}$ \\
\hline & Berro amarillo & $\mathrm{N}$ & $\mathrm{He}$ & $\mathrm{C}$ & $\mathrm{D}$ \\
\hline & Placa & $\mathrm{N}$ & $\mathrm{He}$ & C & $\mathrm{D}$ \\
\hline Portulacaceae & No conocido & $\mathrm{N}$ & $\mathrm{He}$ & $\mathrm{T}$ & $\mathrm{D}$ \\
\hline Haloragaceae & Hierba del sapo & $\mathrm{N}$ & $\mathrm{Hi}$ & $\mathrm{Cr}$ & $\mathrm{D}, \mathrm{S}$ \\
\hline \multirow[t]{2}{*}{ Scrophulariaceae } & Placa roja & $\mathrm{N}$ & $\mathrm{He}$ & $\mathrm{H}$ & $\mathrm{D}$ \\
\hline & Plaquita & $\mathrm{N}$ & $\mathrm{He}$ & $\mathrm{H}$ & $\mathrm{D}$ \\
\hline Poaceae & Cola de zorro & $\mathrm{N}$ & $\mathrm{He}$ & $\mathrm{H}$ & $\mathrm{D}$ \\
\hline Potamogetonaceae & Huiro & $\mathrm{N}$ & $\mathrm{Hi}$ & $\mathrm{Cr}$ & $\mathrm{D}$ \\
\hline Rosaceae & Canelilla & I & $\mathrm{He}$ & $\mathrm{H}$ & $\mathrm{D}, \mathrm{S}$ \\
\hline Campanulaceae & No conocido & $\mathrm{N}$ & $\mathrm{He}$ & $\mathrm{H}$ & $\mathrm{D}$ \\
\hline Poaceae & Pasto azul de marisma & $\mathrm{N}$ & $\mathrm{He}$ & $\mathrm{H}$ & $\mathrm{S}$ \\
\hline \multirow[t]{8}{*}{ Ranunculaceae } & No conocido & $\mathrm{N}$ & $\mathrm{He}$ & $\mathrm{H}$ & $\mathrm{D}$ \\
\hline & Penchaico & $\mathrm{N}$ & $\mathrm{He}$ & $\mathrm{T}$ & $\mathrm{D}, \mathrm{S}$ \\
\hline & Hierba del sapo & $\mathrm{N}$ & $\mathrm{Hi}$ & $\mathrm{Cr}$ & $\mathrm{D}$ \\
\hline & Oreja de gato & $\mathrm{N}$ & $\mathrm{He}$ & $\mathrm{H}$ & $\mathrm{D}$ \\
\hline & Botón de oro chico & $\mathrm{N}$ & $\mathrm{He}$ & $\mathrm{H}$ & $\mathrm{D}$ \\
\hline & Hierba de la vaca & $\mathrm{N}$ & $\mathrm{He}$ & $\mathrm{H}$ & $\mathrm{D}, \mathrm{S}$ \\
\hline & Botón de oro & $\mathrm{N}$ & $\mathrm{He}$ & $\mathrm{H}$ & $\mathrm{D}$ \\
\hline & Botón de oro & I & $\mathrm{He}$ & $\mathrm{H}$ & $\mathrm{D}$ \\
\hline Polygonaceae & Romaza & I & $\mathrm{He}$ & $\mathrm{H}$ & $\mathrm{D}$ \\
\hline Ruppiaceae & Maleza de marisma & $\mathrm{N}$ & $\mathrm{Hi}$ & $\mathrm{Cr}$ & $\mathrm{S}$ \\
\hline \multirow[t]{3}{*}{ Cyperaceae } & Totora & $\mathrm{N}$ & $\mathrm{He}$ & $\mathrm{Cr}$ & $\mathrm{D}, \mathrm{S}$ \\
\hline & Can-Can & $\mathrm{N}$ & $\mathrm{He}$ & $\mathrm{H}$ & $\mathrm{D}$ \\
\hline & Chan-Chan & $\mathrm{N}$ & $\mathrm{He}$ & $\mathrm{H}$ & $\mathrm{D}$ \\
\hline \multirow[t]{3}{*}{ Asteraceae } & No conocido & $\mathrm{N}$ & $\mathrm{He}$ & $\mathrm{H}$ & $\mathrm{D}$ \\
\hline & Hualtata & $\mathrm{N}$ & $\mathrm{He}$ & $\mathrm{H}$ & $\mathrm{D}$ \\
\hline & Lampazo & $\mathrm{N}$ & $\mathrm{He}$ & $\mathrm{H}$ & $\mathrm{D}$ \\
\hline \multirow[t]{3}{*}{ Juncaginaceae } & No conocido & $\mathrm{N}$ & $\mathrm{He}$ & $\mathrm{H}$ & $\mathrm{D}$ \\
\hline & Hierba de la paloma & $\mathrm{N}$ & $\mathrm{He}$ & $\mathrm{H}$ & $\mathrm{S}$ \\
\hline & Hierba de la paloma & $\mathrm{N}$ & $\mathrm{He}$ & $\mathrm{H}$ & $\mathrm{D}, \mathrm{S}$ \\
\hline Valerianaceae & Valeriana & $\mathrm{N}$ & $\mathrm{He}$ & $\mathrm{Cr}$ & $\mathrm{D}$ \\
\hline Scrophulariaceae & No me olvides del campo & I & $\mathrm{He}$ & $\mathrm{H}$ & $\mathrm{D}$ \\
\hline Zannichelliaceae & Cachagüita & $\mathrm{N}$ & $\mathrm{Hi}$ & $\mathrm{Cr}$ & S \\
\hline
\end{tabular}

La ausencia de familia indica que vale la inmediatamente superior. Origen: $\mathrm{N}=$ nativo, $\mathrm{I}=$ introducido. Hábitat: Hi = hidrófitos (plantas acuáticas), $\mathrm{He}=$ helófitos (plantas palustres). Formas de vida: $\mathrm{C}=$ caméfitos, $\mathrm{H}=$ hemicriptófitos, $\mathrm{Cr}=$ criptófitos, $\mathrm{T}=$ terófitos. El género Mimulus se puede considerar parte de la familia Phrymaceae.

Salinidad del agua: $\mathrm{A}=$ dulce, $\mathrm{S}=$ salobre. 
Anexo 2. Presencia de especies de macrófitos en los diferentes ambientes acuáticos. Abreviaturas de los ambientes acuáticos en TABLA 3.

\begin{tabular}{|c|c|c|c|c|c|c|c|c|c|c|c|c|c|c|}
\hline Especie & $\mathrm{R}$ & A & At & $\mathrm{S}$ & $\mathrm{Ar}$ & $\mathrm{Ab}$ & V & $\mathrm{L}$ & $\mathrm{Lb}$ & Lt & $\mathrm{La}$ & $\mathrm{Ch}$ & M & Total \\
\hline Aster vahlii & $\mathrm{X}$ & $\mathrm{X}$ & $\mathrm{X}$ & $\mathrm{X}$ & $\mathrm{X}$ & & $\mathrm{X}$ & $\mathrm{X}$ & $X$ & $\mathrm{X}$ & & & & 9 \\
\hline Polypogon australis & $\mathrm{X}$ & $\mathrm{X}$ & $\mathrm{X}$ & $\mathrm{X}$ & $\mathrm{x}$ & & $\mathrm{x}$ & $\mathrm{x}$ & $\mathrm{x}$ & & & $\mathrm{X}$ & & 9 \\
\hline Juncus microcephalus & $\mathrm{X}$ & $\mathrm{X}$ & & $\mathrm{x}$ & $\mathrm{X}$ & & $\mathrm{x}$ & $\mathrm{x}$ & $\mathrm{x}$ & & & $\mathrm{X}$ & & 8 \\
\hline Holcus lanatus & $\mathrm{X}$ & $\mathrm{X}$ & & $\mathrm{x}$ & $\mathrm{X}$ & & $\mathrm{x}$ & $\mathrm{x}$ & $\mathrm{x}$ & & & & & 7 \\
\hline Hydrocotyle chamaemorus & $\mathrm{X}$ & $\mathrm{X}$ & & $\mathrm{X}$ & $\mathrm{X}$ & & $\mathrm{x}$ & $\mathrm{x}$ & $\mathrm{x}$ & & & & & 7 \\
\hline Juncus procerus & $\mathrm{X}$ & $\mathrm{X}$ & & $\mathrm{X}$ & $\mathrm{X}$ & & $\mathrm{x}$ & $\mathrm{x}$ & $\mathrm{x}$ & & & & & 7 \\
\hline Rumex conglomeratus & $\mathrm{x}$ & $\mathrm{x}$ & & $\mathrm{x}$ & $\mathrm{X}$ & & $\mathrm{x}$ & $\mathrm{x}$ & $\mathrm{x}$ & & & & & 7 \\
\hline Scirpus californicus & $\mathrm{x}$ & $\mathrm{x}$ & & & & & & $\mathrm{x}$ & $\mathrm{X}$ & $\mathrm{X}$ & $\mathrm{X}$ & & $\mathrm{x}$ & 7 \\
\hline Juncus pallescens & $\mathrm{x}$ & $\mathrm{x}$ & & & $\mathrm{X}$ & & $\mathrm{x}$ & $\mathrm{x}$ & $\mathrm{X}$ & & & & & 6 \\
\hline Ranunculus repens & $\mathrm{x}$ & $\mathrm{x}$ & & & $\mathrm{X}$ & & $\mathrm{x}$ & $\mathrm{x}$ & $\mathrm{x}$ & & & & & 6 \\
\hline Veronica anagallis-aquatica & $\mathrm{x}$ & $\mathrm{x}$ & & & $\mathrm{X}$ & & $\mathrm{x}$ & $\mathrm{x}$ & $\mathrm{X}$ & & & & & 6 \\
\hline Blechnum chilense & $\mathrm{x}$ & $\mathrm{x}$ & & $\mathrm{X}$ & $\mathrm{X}$ & & $\mathrm{x}$ & & & & & & & 5 \\
\hline Potamogeton stenostachys & $\mathrm{x}$ & & & & & & & $\mathrm{x}$ & $\mathrm{X}$ & $\mathrm{X}$ & $\mathrm{X}$ & & & 5 \\
\hline Litorella australis & $\mathrm{X}$ & & & & $\mathrm{X}$ & & & $\mathrm{x}$ & $\mathrm{X}$ & & & & & 4 \\
\hline Equisetum bogotense & $\mathrm{X}$ & $\mathrm{X}$ & & & & & $\mathrm{X}$ & $\mathrm{x}$ & & & & & & 4 \\
\hline Carex banksii & $\mathrm{x}$ & $\mathrm{x}$ & & & & & & $\mathrm{x}$ & $\mathrm{X}$ & & & & & 4 \\
\hline Carex darwinii & $\mathrm{x}$ & $\mathrm{x}$ & & & & & & $\mathrm{x}$ & $\mathrm{x}$ & & & & & 4 \\
\hline Carex macloviana & $\mathrm{x}$ & $\mathrm{x}$ & & & & & & $\mathrm{x}$ & $\mathrm{x}$ & & & & & 4 \\
\hline Agrostis stolonifera & $\mathrm{x}$ & & & & & & & $\mathrm{x}$ & $\mathrm{x}$ & & & & & 3 \\
\hline Carex pseudocyperus & $\mathrm{x}$ & & & & & & & $\mathrm{x}$ & $\mathrm{x}$ & & & & & 3 \\
\hline Carex riparia & $\mathrm{x}$ & & & & & & & $\mathrm{x}$ & $\mathrm{X}$ & & & & & 3 \\
\hline Cyperus eragrostis & $\mathrm{X}$ & & & & & & & $\mathrm{x}$ & $\mathrm{X}$ & & & & & 3 \\
\hline Myriophyllum quitense & $\mathrm{x}$ & & & & & & & $\mathrm{x}$ & $\mathrm{x}$ & & & & & 3 \\
\hline Glyceria multiflora & $\mathrm{x}$ & $\mathrm{X}$ & & & & & & & $\mathrm{x}$ & & & & & 3 \\
\hline Senecio smithii & $\mathrm{x}$ & $\mathrm{x}$ & & & & & & & $\mathrm{X}$ & & & & & 3 \\
\hline Gunnera magellanica & & & & $\mathrm{x}$ & $\mathrm{X}$ & & $\mathrm{x}$ & & & & & & & 3 \\
\hline Potentilla anserina & & & & & $\mathrm{X}$ & & & & & & & $\mathrm{X}$ & $\mathrm{X}$ & 3 \\
\hline Pratia repens & & & & & $\mathrm{x}$ & & & $\mathrm{x}$ & & & & $\mathrm{X}$ & & 3 \\
\hline Ranunculus uniflorus & & & & & & & & & $\mathrm{X}$ & & & $\mathrm{X}$ & $\mathrm{X}$ & 3 \\
\hline Senecio fistulosus & $\mathrm{x}$ & $\mathrm{x}$ & & & & & & & & & & & & 2 \\
\hline Limosella australis & $\mathrm{x}$ & & & & $\mathrm{X}$ & & & & & & & & & 2 \\
\hline Lilaeopsis macloviana & $\mathrm{x}$ & & & & & & & $\mathrm{X}$ & & & & & & 2 \\
\hline Anagallis alternifolia & $\mathrm{x}$ & & & & & & & & $\mathrm{X}$ & & & & & 2 \\
\hline Triglochin striata & $\mathrm{X}$ & & & & & & & & & & & & $\mathrm{X}$ & 2 \\
\hline Baccharis sagittalis & & $\mathrm{X}$ & $\mathrm{X}$ & & & & & & & & & & & 2 \\
\hline Geum magellanicum & & $\mathrm{x}$ & & & $\mathrm{X}$ & & & & & & & & & 2 \\
\hline Callitriche palustris & & $\mathrm{x}$ & & & & & & & $\mathrm{X}$ & & & & & 2 \\
\hline Scirpus cernuus & & & $\mathrm{X}$ & & & $\mathrm{X}$ & & & & & & & & 2 \\
\hline Calceolaria tenella & & & & & $\mathrm{X}$ & & $\mathrm{X}$ & & & & & & & 2 \\
\hline Caltha sagittata & & & & & $\mathrm{x}$ & & $\mathrm{x}$ & & & & & & & 2 \\
\hline Cardamine glacialis & & & & & $\mathrm{x}$ & & $\mathrm{x}$ & & & & & & & 2 \\
\hline Epilobium australe & & & & & $\mathrm{X}$ & & $\mathrm{X}$ & & & & & & & 2 \\
\hline Epilobium ciliatum & & & & & $\mathrm{x}$ & & $\mathrm{x}$ & & & & & & & 2 \\
\hline Lobelia alata & & & & & $\mathrm{X}$ & & $\mathrm{x}$ & & & & & & & 2 \\
\hline Ourisia coccinea & & & & & $\mathrm{X}$ & & $\mathrm{x}$ & & & & & & & 2 \\
\hline Ourisia ruelloides & & & & & $\mathrm{X}$ & & $\mathrm{x}$ & & & & & & & 2 \\
\hline Carex curta & & & & & $\mathrm{x}$ & & & & $\mathrm{X}$ & & & & & 2 \\
\hline Lilaea scilloides & & & & & $\mathrm{X}$ & & & & $\mathrm{X}$ & & & & & 2 \\
\hline
\end{tabular}


Ranunculus cymbalaria

Ranunculus minutiflorus

Ranunculus peduncularis

Ranunculus pseudotrullifolius

Juncus planifolius

Juncus stipulatus

Valeriana lapathifolia

Eleocharis pachycarpa

Isoetes savatierii

Ranunculus biternatus

Eleocharis melanostachys

Scirpus inundatus

Senecio acanthifolius

Cardamine bonariensis

Mimulus parviflorus

Mimulus glabratus

Mimulus luteus

Dysopsis glechomoides

Gunnera tinctoria

Azolla filiculoides

Callitriche lechleri

Callitriche antarctica

Callitriche terrestris

Hippuris vulgaris

Lemna minima

Lycopus europaeus

Ranunculus aquatilis

Marsippospermum grandiflorum

Tetroncium magellanicum

Crassula peduncularis

Juncus bufonius .

Lythrum portula

Montia fontana

Cotula coronopifolia

Puccinellia glaucescens

Ruppia filifolia

Triglochin concinna

Zannichellia palustris

Total (86 especies)
$X$
$X$
$X$
$X$
$X$
$X$

X X

$\mathrm{X} \quad \mathrm{X}$

$X \quad X$

$X \quad X$

X X

$\mathrm{X}$

$\mathrm{X}$

$\mathrm{X}$

$\mathrm{X}$

$\mathrm{X}$

$\mathrm{X}$

X

X

X

$\mathrm{X}$

$\mathrm{X}$

$\mathrm{X}$

$\mathrm{X}$

$\mathrm{X}$

$\mathrm{X}$

$\mathrm{X}$

$\mathrm{X}$

$\mathrm{X}$

X

X

2

2

2

2

2

X 2

2

2

2

2

2

1

1

1

1

1

1

1

1

1

1

1

1

1

1

1

$\begin{array}{ll}X & 1\end{array}$

$\begin{array}{ll}\mathrm{X} & 1 \\ \mathrm{X} & 1\end{array}$

$\begin{array}{ll}X & 1\end{array}$

$\begin{array}{ll}\mathrm{X} & 1\end{array}$

$\begin{array}{ll}X & 1\end{array}$

X 1

X 1

$\begin{array}{ll}\mathrm{X} & 1\end{array}$

X 1 
